Accepted for publication in The Astrophysical Journal

Preprint typeset using $\mathrm{LATEX}_{\mathrm{E}}$ style emulateapj v. 5/2/11

\title{
TWO NEW LONG-PERIOD HOT SUBDWARF BINARIES WITH DWARF COMPANIONS*
}

\author{
Brad N. Barlow ${ }^{\dagger \dagger}$, SAndra E. Liss ${ }^{2}$, Richard A. WAdE ${ }^{1}$, and Elizabeth M. GREen ${ }^{3}$ \\ ${ }^{1}$ Dept of Astronomy and Astrophysics, The Pennsylvania State University, 525 Davey Lab, University Park, PA 16802, USA \\ ${ }^{2}$ Department of Astronomy, University of Virginia, P.O. Box 400325, Charlottesville, VA 22904-4325, USA \\ ${ }^{3}$ Steward Observatory, University of Arizona, 933 N. Cherry Avenue, Tucson, AZ 85721, USA \\ Accepted for publication in The Astrophysical Journal
}

\begin{abstract}
Hot subdwarf stars with $\mathrm{F}-\mathrm{K}$ main sequence binary companions have been known for decades, but the first orbital periods for such systems were published just recently. Current observations suggest that most have long periods, on the order of years, and that some are or once were hierarchical triple systems. As part of a survey with the Hobby-Eberly Telescope, we have been monitoring the radial velocities of several composite-spectra binaries since 2005 in order to determine their periods, velocities, and eccentricities. Here we present observations and orbital solutions for two of these systems, PG 1449+653 and PG 1701+359. Similar to the other sdB+F/G/K binaries with solved orbits, their periods are long, $909 \mathrm{~d}$ and $734 \mathrm{~d}$, respectively, and pose a challenge to current binary population synthesis models of hot subdwarf stars. Intrigued by their relatively large systemic velocities, we also present a kinematical analysis of both targets and find that neither is likely a member of the Galactic thin disk.

Subject headings: binaries: spectroscopic — ephemerides — subdwarfs — techniques: radial velocities
\end{abstract}

\section{INTRODUCTION}

Mystery has shrouded the formation of hot subdwarf stars since they were first recognized as a blue extension of the horizontal branch in the Galactic halo by Greenstein \& Sargent (1974) almost forty years ago. Since then, they have been found in all Galactic populations (Altmann et al. 2004; Napiwotzki 2008), globular clusters, and even the elliptical galaxy M32 (Brown et al. 2000). In this regard, it is interesting to note that the known hot subdwarfs seem to occur only in rather old populations, with ages greater than about 4-5 Gyr, and are much more common (as a percentage of the core Heburning stars) in populations with high metallicity, e.g., the old, metal-rich open clusters NGC 188 and NGC 6791 (Liebert et al. 1994; Green et al. 2006).

Models show hot subdwarf B (sdB) stars are core Heburning stars surrounded by extremely thin $\mathrm{H}$ envelopes, with masses around $0.5 \mathrm{M}_{\odot}$ and radii near $0.2 \mathrm{R}_{\odot}$ Heber 1986; Saffer et al. 1994). To explain their lack of hydrogen (in contrast to other core helium-burning stars), various single-star and binary-evolution formation scenarios have been constructed, all of which entail the sdB progenitor losing its outer hydrogen envelope while on or near the red giant branch (RGB). As single-star scenarios are ad hoc, binary-star hypotheses have generally been favored to explain the loss of the $\mathrm{H}$ envelope, following early theoretical work by Mengel et al. (1976).

Han et al. (2002, 2003) presented the first results from a major binary population synthesis (BPS) study of hot subdwarf stars and described five primary formation channels, including two common envelope (CE) channels, two stable Roche lobe overflow (RLOF) channels, and the

\footnotetext{
* Based on observations obtained with the Hobby-Eberly Telescope, which is a joint project of the University of Texas at Austin, the Pennsylvania State University, Stanford University Ludwig-Maximilians-Universität München, and Georg-August-

Universität Göttingen.

$\dagger$ bbarlow@psu.edu
}

merger of a He--core white dwarf binary. Other notable studies include those of Clausen \& Wade (2011), who demonstrate that the merger of a He-core white dwarf and M dwarf can create an sdB, and Nelemans (2010), who put forth an alternative CE channel employing the $\gamma$-formalism. Many assumptions influence the outcomes in BPS models, including the common-envelope ejection efficiency, minimum mass for core He ignition, and envelope binding energy. Changing any one of these parameters, even moderately, can greatly alter the hot subdwarf population predicted by the codes. Clausen et al. (2012) recently demonstrated this sensitivity using a grid of BPS models with different inputs. They found that (1) a wide range of parameter sets can reproduce the observed subpopulation of short-period white dwarf and $\mathrm{M}$ dwarf binaries, which are products of CE evolution, and (2) observations of systems with $\mathrm{F}-\mathrm{K}$ main sequence (MS) companions are needed to constrain the physics governing RLOF processes.

While there are numerous short-period orbits (days) published for post-CE sdB binaries (see Table A.1 of Geier et al. 2011), no definitive periods under 30 d have been reported for systems with MS companions earlier than spectral type $\mathrm{M} 3$ On the contrary, long-term monitoring of these systems (Deca et al. 2012; Østensen \& Van Winckel 2012; Barlow et al.|2012; Vos et al. 2012) has shown their periods are on the order of years, as originally suspected by Saffer et al. (2001) more than a decade ago. Such long periods appear to be inconsistent with the predictions of Han et al. (2002, 2003) and pose a major challenge to BPS models. Of course, orbital parameters for many more long-period systems are needed before any definitive conclusions can be drawn.

In 2005 we began monitoring the radial velocities of

3 Moni Bidin \& Piotto (2010) suggest that the star M5865 in globular cluster NGC 6752 is a hot subdwarf + G-K MS binary with a period of order $5 \mathrm{~d}$, but details have not been published. 
15 hot subdwarf stars with $\mathrm{F}-\mathrm{K}$ main sequence companions at the Hobby-Eberly Telescope (HET) in order to constrain their orbital parameters. Details of this study may be found in Barlow et al. 2012 (hereafter, Paper I). Although we could only solve for the orbits of three systems using our initial data set, we presented preliminary periods for 12 targets. Almost all have periods in excess of 100 days; only two appear to have short periods, and these targets are likely hierarchical triple- or quadruple-star systems. Our preliminary results confirm previous findings that composite-spectra sdB systems tend to have long periods. To solve for the orbits of the remaining systems, we began collecting follow-up observations with the High Resolution Spectrograph on the HET in Mar 2012. An up-to-date orbital period histogram of all hot subdwarf binaries (Fig. 5 of Paper I) shows a clear dichotomy between systems with F-K main sequence companions $(\mathrm{P}>100 \mathrm{~d})$ and those with white dwarf or $\mathrm{M}$ dwarf companions $(\mathrm{P}<100 \mathrm{~d})$. A possible period gap is emerging around $\mathrm{P} \sim 100 \mathrm{~d}$, but the statistics are presently too poor to claim this feature as real.

Here we present new spectroscopic observations of the $\mathrm{sdB}+\mathrm{MS}$ binaries $\mathrm{PG} 1449+653(\mathrm{~V}=13.6)$ and $\mathrm{PG}$ $1701+359(\mathrm{~V}=13.2)$. We find orbital periods exceeding two years for both targets, a result in line with other $\mathrm{sdB}+\mathrm{F} / \mathrm{G} / \mathrm{K}$ binaries. Intrigued by their relatively large systemic velocities, we combine our spectroscopy with proper motion measurements to calculate Galactic orbits and find that neither system is likely a member of the Galactic thin disk.

\section{OBSERVATIONS \& REDUCTIONS}

From 2005 to 2008 we monitored PG $1449+653$ and PG $1701+359$ spectroscopically with the MediumResolution Spectrograph (MRS; Ramsey et al. 1998) on the Hobby-Eberly Telescope (HET). Full details on the observational setup used may be found in Paper I. We commenced follow-up observations in 2012 with the High Resolution Spectrograph (HRS) on the HET, using the $2^{\prime \prime}$ optical fiber pair, the $316 \mathrm{~mm}^{-1}$ cross-disperser grating, and $2 \times 3$ on-chip binning to achieve an average resolution of $\mathrm{R}=30,000$ over the $4076-7838 \AA$ spectral range. This configuration yields an average dispersion of $\sim 3.6$ $\mathrm{km} \mathrm{s}^{-1}$ per binned pixel and samples $\sim 2.7$ pixels per resolution element. Target spectra were taken in pairs and later combined to help remove cosmic rays. Standard calibration frames were also collected each night using the same instrumental setup, including twilight sky spectra, bias frames, quartz lamp flatfield spectra, and, on most occasions, spectra of telluric, RV, and spectrophotometric standard stars.

We bias-subtracted and flat-fielded all HET data using the $c c d p r o c$ routine in IRAFA and optimally extracted apertures with the apall function. Some echelle orders were discarded due to CCD fringing, heavy contamination from sky emission and telluric absorption features, cross-disperser order overlap, and low throughput. For HRS (MRS), we kept a total of 48 (14) orders covering

4 IRAF is distributed by the National Optical Astronomy Observatories, which are operated by the Association of Universities for Research in Astronomy, Inc., under cooperative agreement with the National Science Foundation
TABLE 1

Summary of SPECtroscopic ObSERVATIONS

\begin{tabular}{llll}
\hline \hline Target & Telescope/Instrum. & $\mathrm{N}_{\text {obs }}$ & Year \\
\hline PG 1449+653 & HET/HRS & 7 & $2012-2013$ \\
& HET/MRS & 8 & $2005-2008$ \\
& MMT/Blue Spect. & 5 & 1997 \\
PG 1701+359 & HET/HRS & 8 & $2012-2013$ \\
& HET/MRS & 13 & $2005-2008$ \\
& MMT/Blue Spect. & 8 & $1997,2002-2003$ \\
\hline
\end{tabular}

4100-7000 $\AA(4400-6200 \AA)$.

We supplement the HET data with single-order, longslit spectra from the Blue Spectrograph at the Multi Mirror Telescope (MMT) obtained in 1997 and from 2002 to 2003. An $832 \mathrm{~mm}^{-1}$ grating was used in second order to achieve wavelength coverage of $4000-4950 \AA$ at a resolution of $1.1 \AA(\mathrm{R}=4100)$ and dispersion near $0.36 \AA$ pixel $^{-1}$. MMT data were reduced using the same IRAF routines mentioned above for the HET observations. Table 1 summarizes the entire data set.

\section{ANALYSIS}

We generally followed the same procedures outlined in Paper I to measure radial velocities (RVs) and determine the best-fitting orbital parameters for each system; we highlight only certain aspects of these procedures here (along with any deviations from Paper I) and refer readers to Paper I for full disclosure of our analysis methods.

\subsection{Companion classification}

We cross-correlated the PG $1449+653$ and PG $1701+359$ spectra with MS standards (F0-K7) observed by the MMT and HET in the same instrumental configurations as our data. IRAF's fxcor routine was used for all regions of the spectra except those around the hydrogen Balmer and helium lines. We determined the bestmatching MS spectra for each target from the TonryDavis ratio (R-value output from fxcor; Tonry \& Davis 1979) and the fxcor-outputted velocity errors for each template. A spectral type of G0V was consistently found for PG $1449+653$. The PG $1701+359$ spectrum, which has lower $\mathrm{S} / \mathrm{N}$ and higher dilution, was best-matched to a K0V standard, although several G-type standards (as early as G0V) also fit the spectra reasonably well.

We also adopt the approach used by Stark \& Wade (2003) to photometrically determine the spectral types of the cool companions. After correcting for reddening (Schlegel et al. 1998), we compare the colors of PG $1449+653$ and PG $1701+359$ to a theoretical grid of $B-V$ and $J-K$ colors for hot subdwarf binaries with cool main sequence companions. We note that Population I colors were assumed for the cool companions, although kinematics suggest these systems might have halo-like orbits (\$5). For each pairing, the dilution factor of the companion spectrum in the $V$-band $\left(D_{V} \equiv L_{\text {comp }} / L_{\text {total }}\right)$ was varied from $1-100 \%$. Color indices for the cool companions and hot subdwarfs are taken from Johnson (1966) and Stark \& Wade (2003), respectively. For PG $1449+653$, we find colors consistent with a 'typical' hot subdwarf star and a F8-G3 dwarf (best fit: G1) with a dilution factor around $D_{V} \sim 0.3$; these results agree with the spectroscopically-determined value. PG $1701+359$ 's 
TABLE 2

Heliocentric Radial Velocities of the Cool Companion

\begin{tabular}{|c|c|c|c|c|c|}
\hline $\begin{array}{c}\text { HJD } \\
-2450000\end{array}$ & $\begin{array}{c}\mathrm{RV}_{\mathrm{MS}} \\
{\left[\mathrm{km} \mathrm{s}^{-1}\right]}\end{array}$ & $\overline{\text { Facility }}$ & $\begin{array}{c}\text { HJD } \\
-2450000\end{array}$ & $\begin{array}{c}\mathrm{RV}_{\mathrm{MS}} \\
{\left[\mathrm{km} \mathrm{s}^{-1}\right]}\end{array}$ & $\overline{\text { Facility }}$ \\
\hline \multicolumn{3}{|c|}{ PG $1449+653$} & \multicolumn{3}{|c|}{ PG $1701+359$} \\
\hline 511.0187 & $-128.4 \pm 1.0$ & MMT & 627.8929 & $-121.0 \pm 1.9$ & MMT \\
\hline 626.8101 & $-129.3 \pm 1.3$ & MMT & 633.8716 & $-123.3 \pm 1.9$ & MMT \\
\hline 627.7691 & $-129.1 \pm 1.0$ & MMT & 642.8422 & $-122.2 \pm 1.9$ & MMT \\
\hline 642.7782 & $-129.3 \pm 1.0$ & MMT & 643.7559 & $-124.8 \pm 1.8$ & MMT \\
\hline 701.6427 & $-130.6 \pm 1.4$ & MMT & 701.6934 & $-122.9 \pm 2.0$ & MMT \\
\hline 3462.8508 & $-136.0 \pm 0.8$ & HET/MRS & 1654.7222 & $-119.6 \pm 1.1$ & MMT \\
\hline 3479.8705 & $-136.4 \pm 1.0$ & HET/MRS & 2544.6458 & $-123.0 \pm 1.7$ & MMT \\
\hline 3503.8035 & $-138.0 \pm 0.9$ & HET/MRS & 2826.8606 & $-123.9 \pm 1.6$ & MMT \\
\hline 3520.6751 & $-138.4 \pm 0.8$ & HET/MRS & 3482.8107 & $-122.2 \pm 1.2$ & HET/MRS \\
\hline 3758.0254 & $-143.5 \pm 0.7$ & HET/MRS & 3520.9447 & $-122.4 \pm 0.9$ & HET/MRS \\
\hline 3827.9095 & $-138.4 \pm 1.1$ & HET/MRS & 3543.8831 & $-123.8 \pm 1.3$ & HET/MRS \\
\hline 3855.8400 & $-138.3 \pm 1.2$ & HET/MRS & 3584.7790 & $-123.6 \pm 1.1$ & $\mathrm{HET} / \mathrm{MRS}$ \\
\hline 4216.8374 & $-128.5 \pm 0.8$ & HET/MRS & 3609.7073 & $-122.8 \pm 1.6$ & HET/MRS \\
\hline 5991.9222 & $-128.4 \pm 0.2$ & HET/HRS & 3827.8767 & $-120.4 \pm 1.3$ & HET/MRS \\
\hline 6018.8438 & $-128.5 \pm 0.2$ & HET/HRS & 3854.7918 & $-119.0 \pm 1.3$ & HET/MRS \\
\hline 6061.6989 & $-129.5 \pm 0.2$ & $\mathrm{HET} / \mathrm{HRS}$ & 4273.8819 & $-122.1 \pm 1.2$ & HET/MRS \\
\hline 6086.6883 & $-130.2 \pm 0.2$ & HET/HRS & 4506.0149 & $-121.3 \pm 0.9$ & HET/MRS \\
\hline 6118.6349 & $-131.1 \pm 0.2$ & HET/HRS & 4569.8307 & $-117.1 \pm 2.9$ & HET/MRS \\
\hline 6334.9466 & $-142.6 \pm 0.2$ & $\mathrm{HET} / \mathrm{HRS}$ & 4582.7928 & $-118.8 \pm 0.8$ & HET/MRS \\
\hline 6376.9266 & $-144.2 \pm 0.2$ & HET/HRS & 4612.7189 & $-117.1 \pm 1.5$ & HET/MRS \\
\hline$\ldots$ & $\ldots$ & $\ldots$ & 4671.7919 & $-115.3 \pm 1.4$ & HET/MRS \\
\hline$\ldots$ & $\ldots$ & $\ldots$ & 5997.8963 & $-121.0 \pm 0.4$ & $\mathrm{HET} / \mathrm{HRS}$ \\
\hline$\ldots$ & $\ldots$ & $\ldots$ & 6040.7881 & $-119.9 \pm 0.2$ & HET/HRS \\
\hline$\ldots$ & $\ldots$ & $\ldots$ & 6068.9367 & $-118.7 \pm 0.3$ & HET/HRS \\
\hline$\ldots$ & $\ldots$ & $\ldots$ & 6098.6339 & $-117.6 \pm 0.2$ & HET/HRS \\
\hline$\ldots$ & $\ldots$ & $\ldots$ & 6145.7336 & $-116.9 \pm 0.2$ & HET/HRS \\
\hline$\ldots$ & $\ldots$ & $\ldots$ & 6191.6229 & $-116.2 \pm 0.3$ & HET/HRS \\
\hline$\ldots$ & $\ldots$ & $\ldots$ & 6326.0072 & $-118.3 \pm 0.3$ & HET/HRS \\
\hline$\ldots$ & $\ldots$ & $\ldots$ & 6370.8962 & $-119.5 \pm 0.3$ & HET/HRS \\
\hline
\end{tabular}

composite colors imply a G7-K2 dwarf companion (best fit: K0) with $D_{V} \sim 0.15$, also consistent with the observed composite spectrum.

\subsection{Radial velocity measurements}

Heliocentric radial velocities were determined by crosscorrelating each spectrum against velocity standards using IRAF's fxcor and rvcorrect routines. All MMT spectra were correlated against the velocity standard HD 39587, a G0V dwarf, which was observed using the same instrumental setup as our target observations. For the HET data, we achieve the best results when using a single, high $\mathrm{S} / \mathrm{N}$ twilight sky spectrum as the correlation template for all HRS and MRS observations. We extracted HET velocities from each order individually, being sure to avoid spectral regions where subdwarf features compromise our ability to measure the cool companion velocities. We adopt as the error the standard deviation of the mean of the order-by-order velocity fits. Typical velocity errors ranged from $1-1.5 \mathrm{~km} \mathrm{~s}^{-1}$ for MRS, 200-400 m s${ }^{-1}$ for HRS, and 1-1.5 km s${ }^{-1}$ for the MMT spectra. Table 2 presents all cool companion velocities and their associated errors.

We attempted to measure the orbital reflex motion of the hot subdwarf in each system by examining the positions of He I \& He II absorption profiles. We avoided using the sdB's H Balmer lines as they are contaminated by narrower Balmer lines from the cool companion (and
TABLE 3

Heliocentric Radial Velocities OF THE SDB IN PG $1449+653$

\begin{tabular}{ccc}
\hline \hline $\begin{array}{c}\text { HJD } \\
-2450000\end{array}$ & $\begin{array}{c}\mathrm{RV}_{\mathrm{MS}} \\
{\left[\mathrm{km} \mathrm{s}^{-1}\right]}\end{array}$ & Facility \\
\hline 3462.8508 & $-129.9 \pm 5.0$ & HET/MRS \\
3479.8705 & $-130.5 \pm 6.0$ & HET/MRS \\
3503.8035 & $-130.3 \pm 5.6$ & HET/MRS \\
3520.6751 & $-124.5 \pm 5.0$ & HET/MRS \\
3758.0254 & $-122.3 \pm 3.9$ & HET/MRS \\
3827.9095 & $-122.5 \pm 6.6$ & HET/MRS \\
3855.8400 & $-128.3 \pm 7.2$ & HET/MRS \\
4216.8374 & $-135.3 \pm 4.7$ & HET/MRS \\
5991.9222 & $-146.9 \pm 3.3$ & HET/HRS \\
6018.8438 & $-142.5 \pm 2.9$ & HET/HRS \\
6061.6989 & $-144.4 \pm 3.8$ & HET/HRS \\
6086.6883 & $-140.8 \pm 2.9$ & HET/HRS \\
6118.6349 & $-137.7 \pm 2.9$ & HET/HRS \\
6334.9466 & $-120.2 \pm 3.0$ & HET/HRS \\
6376.9266 & $-119.9 \pm 3.1$ & HET/HRS \\
\hline
\end{tabular}

in the MRS/HRS spectra, they are broader than the wavelength coverage of individual echelle orders). For PG $1449+653$, we extracted RVs by cross-correlating the He I $5875 \AA$ profile in each spectrum against a self template, chosen to be the highest $\mathrm{S} / \mathrm{N}$ spectrum obtained. The template's zero point was determined by cross- 

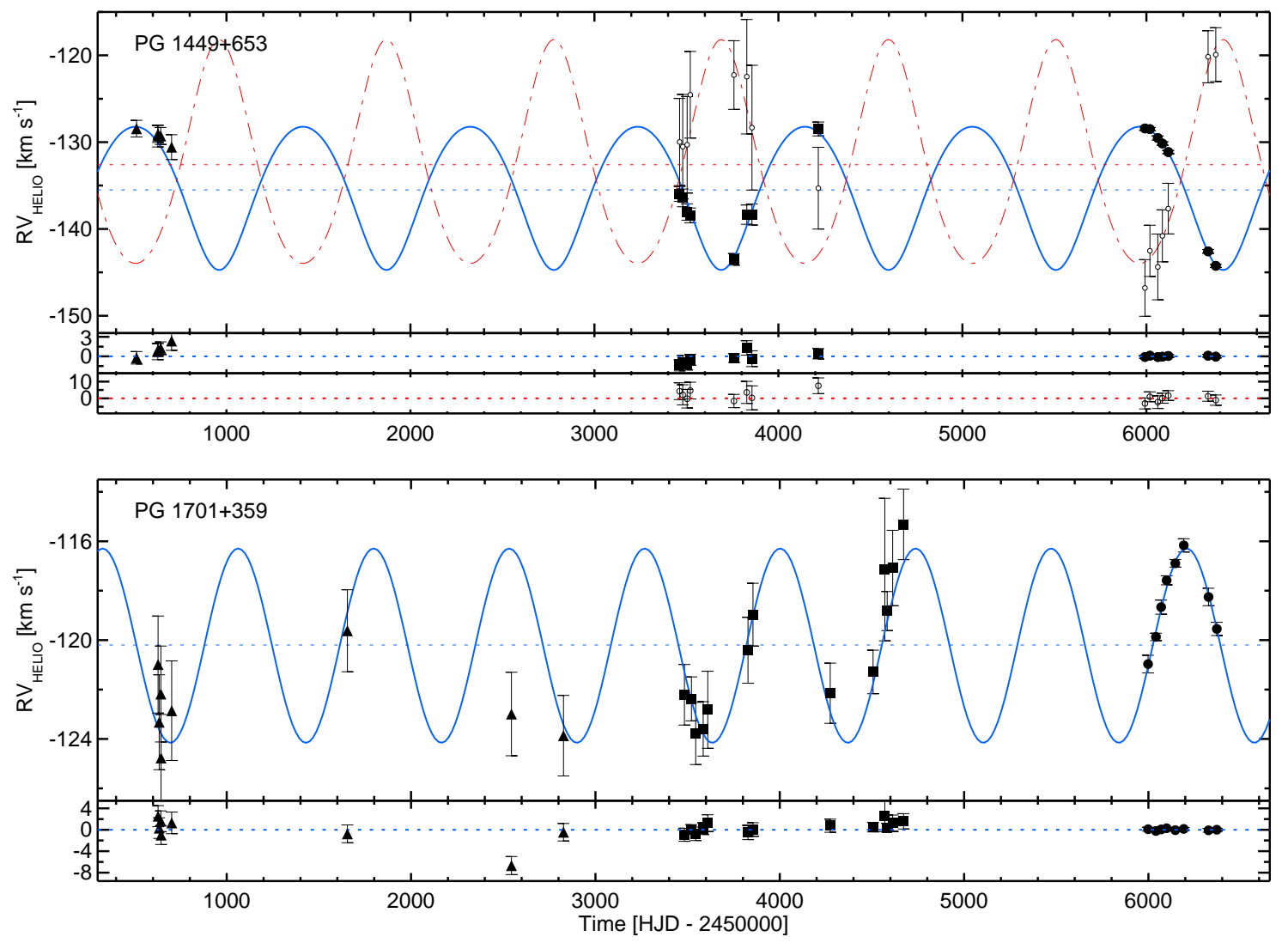

FIG. 1. - Heliocentric radial velocities of PG 1449+653 (top panel) and PG 1701+359 (bottom panel). Cool companion measurements from HET/MRS, HET/HRS, and MMT/Blue Spectrograph are shown with filled circles, squares, and triangles, respectively. Open circles in the top panel mark the sdB velocities measured from He lines in the HET spectra. Some of the error bars are difficult to see as they are smaller than the symbols. The best-fitting circular orbital solutions for the sdB and cool companion are denoted with dot-dashed (orange) and solid (blue) lines, respectively. Residuals from this fit are shown in the lower portions of the panels for the cool companion and, for PG 1449+653, the hot subdwarf. (A color version of this figure is available in the online journal.)

correlating the template against an sdB model spectrum with $\mathrm{T}_{\text {eff }}=30000 \mathrm{~K}$ and $\log g=5.58$. This synthetic template includes pressure broadening and asymmetry in the $5875 \AA$ He I multiplet. Table 3 summarizes the sdB velocities measured from all HET spectra. Unfortunately, our efforts to extract precise $\mathrm{sdB}$ velocities were futile in the case of PG $1701+359$. This shortcoming likely results from a combination of a lower $\mathrm{S} / \mathrm{N}$ in many of the spectra (due to shorter exposure times) and intrinsically weaker He I lines, which could result from the sdB in PG 1701+359 having a significantly different photospheric helium abundance and/or effective temperature than the sdB in $\mathrm{PG} 1449+653$. We also note that the orbital velocities associated with PG 1701+359 are more than a factor of two smaller than those of PG $1449+653$ and, naturally, harder to detect.

\subsection{Determining the orbital parameters}

We solved for the full set of orbital parameters $(P$, $\left.K, e, \omega, \gamma, T_{0}\right)$ using the IDL-based RVLIN software (Wright \& Howard 2009) and estimated parameter uncertainties using a RVLIN-drive bootstrapping technique (Wang et al. 2012). After first running the code with eccentricity $(e)$ fixed to zero, we refitted the data with it and the argument of periapsis $(\omega)$ left adjustable. We also allowed RVLIN to freely fit any zero-point offsets between the MMT, MRS/HET, and HRS/HET velocities, but as no significant shifts were found, we ultimately set the offsets to zero. To be certain RVLIN converged on the global minimum, we computed a 'floating-mean periodogram' (Cumming et al. 1999) for each RV curve to investigate $\chi^{2}$ as a function of period. During this process, a series of sine waves are fitted to the data with adjustable amplitudes and periods ranging from 0.1 to $10000 \mathrm{~d}$ (in steps of $\log P[\mathrm{~d}]=0.001$ ). Only one probable period emerges from the periodogram for each target; the next-largest alias peaks are $10^{3}$ times less probable. These periods also correspond to the global minima found by RVLIN, and so we are confident we have found the correct periods.

\section{THE ORBITAL SOLUTIONS}

Figure 11 and Table 4 present the velocity curves and best-fitting orbital parameters for PG $1701+359$ and PG $1449+643$, which we discuss in detail below.

\section{1. $P G 1449+653$}

The abundance of absorption features from the early $\mathrm{G}$ dwarf provided robust RV measurements with mean errors ranging from $245 \mathrm{~m} \mathrm{~s}^{-1}$ (HRS/HET) to $1400 \mathrm{~m} \mathrm{~s}^{-1}$ (MMT). Measuring the reflex motion of the sdB proved more difficult as most of the subdwarf's absorption features (H Balmer lines, He I lines) were heavily contaminated by features from the cool companion and could not provide reliable velocity estimates. We were only able to 
TABLE 4

Orbital Parameters

\begin{tabular}{|c|c|c|c|c|c|c|c|c|c|c|c|c|c|}
\hline \multirow{2}{*}{$\begin{array}{c}\text { Target } \\
\text { PG } 1449+653\end{array}$} & \multicolumn{2}{|c|}{$\begin{array}{c}P \\
{[\mathrm{~d}]}\end{array}$} & \multicolumn{2}{|c|}{$\begin{array}{c}T_{0}{ }^{a} \\
\text { [HJD-2450000] }\end{array}$} & $e$ & \multicolumn{2}{|c|}{$\begin{array}{c}\omega \\
{[\mathrm{deg}]} \\
\end{array}$} & \multicolumn{2}{|c|}{$\begin{array}{c}K_{\mathrm{MS}} \\
{\left[\mathrm{km} \mathrm{s}^{-1}\right]}\end{array}$} & \multicolumn{2}{|c|}{$\begin{array}{c}K_{\mathrm{sdB}} \\
{\left[\mathrm{km} \mathrm{s}^{-1}\right]}\end{array}$} & \multicolumn{2}{|c|}{$\begin{array}{c}\gamma \\
{\left[\mathrm{km} \mathrm{s}^{-1}\right]}\end{array}$} \\
\hline & 909 & \pm 2 & 3675 & \pm 35 & $0.11 \pm 0.02$ & 174 & \pm 15 & 8.2 & \pm 0.3 & 12.8 & \pm 1.1 & -135.5 & \pm 0.2 \\
\hline & 908 & \pm 2 & 3251 & \pm 40 & 0.0 (fixed) & & $\ldots$ & 8.3 & \pm 0.2 & 13.5 & \pm 0.8 & -136.4 & \pm 0.2 \\
\hline \multirow[t]{2}{*}{ PG $1701+359$} & 738 & \pm 4 & 3869 & \pm 80 & $0.07 \pm 0.04$ & 298 & \pm 38 & 3.6 & \pm 0.2 & & .. & -120.1 & \pm 0.2 \\
\hline & 734 & \pm 3 & 3269 & \pm 60 & 0.0 (fixed) & & $\ldots$ & 3.9 & \pm 0.2 & & ... & -120.2 & \pm 0.2 \\
\hline
\end{tabular}

TABLE 5

Coordinates, Velocities, and Distance Estimates

\begin{tabular}{ccccccc}
\hline \hline Target & $\begin{array}{c}\mathrm{RA} \\
{[\mathrm{J} 2000]}\end{array}$ & $\begin{array}{c}\text { Dec } \\
{[\mathrm{J} 2000]}\end{array}$ & $\begin{array}{c}\mu_{\alpha} \cos \delta^{a} \\
{\left[\mathrm{mas} \mathrm{yr}^{-1}\right]}\end{array}$ & $\begin{array}{c}\mu_{\delta}^{a} \\
{\left[\mathrm{mas} \mathrm{yr}^{-1}\right]}\end{array}$ & $\begin{array}{c}\gamma \\
{\left[\mathrm{km} \mathrm{s}^{-1}\right]}\end{array}$ & $\begin{array}{c}\mathrm{d}^{b} \\
{[\mathrm{kpc}]}\end{array}$ \\
\hline PG 1449+653 & $14: 50: 36.1$ & $+65: 05: 53$ & $-21.7 \pm 2.1$ & $13.6 \pm 1.0$ & $-135.5 \pm 0.2$ & $0.98 \pm 0.16$ \\
PG 1701+359 & $17: 03: 21.6$ & $+35: 48: 49$ & $-57.9 \pm 4.0$ & $20.4 \pm 0.9$ & $-120.2 \pm 0.2$ & $0.69 \pm 0.15$ \\
\hline PG 1104+243 & $11: 07: 26.3$ & $+24: 03: 12$ & $-65.9 \pm 1.2$ & $-25.1 \pm 1.2$ & $-15.68 \pm 0.05$ & $0.33 \pm 0.11$ \\
PG 1317+123 & $13: 19: 53.6$ & $+12: 03: 59$ & $-6.9 \pm 1.1$ & $-1.6 \pm 1.1$ & $+40.3 \pm 0.2$ & $0.33 \pm 0.11$ \\
PG 1338+611 & $13: 40: 14.7$ & $+60: 52: 48$ & $14.5 \pm 0.9$ & $-61.4 \pm 0.8$ & $+32.58 \pm 0.07$ & $0.32 \pm 0.11$ \\
\hline${ }^{a}$ proper motions taken from UCAC4 (Zacharias et al. 2012) & & & \\
${ }^{b}$ calculated from the distance modulus assuming a 'typical' sdB star and our best estimate of the \\
companion's spectral type; error bars are conservative.
\end{tabular}

extract velocities from the HET data, using the $5875 \AA$ He I line alone. This feature, which is significantly diluted due to continuum from the companion, appears in two adjacent orders in the MRS spectra, but only in one order in the HRS data. Using the methods described in $\S 3.2$, we were able to extract sdB RVs with errors ranging from $3-7 \mathrm{~km} \mathrm{~s}^{-1}$.

The top panel of Figure 1 presents the MS and sdB velocity curves, which are 180 degrees out of phase, as expected for a binary system. Given the relatively large uncertainties on the sdB measurements, we used only the MS velocities to determine the orbital parameters with RVLIN. Table 4 lists the results for the best-fitting circular and elliptical orbits. To determine whether the eccentric solution is preferred, we apply the revised LucySweeney (LS) test (Lucy 2012; Lucy \& Sweeney 1971), which uses Bayes' theorem to determine bounds on the exact eccentricity using the value $e \pm \mu$ we measured from RVLIN. According to the LS test, we can reject the circular orbit solution in favor of an eccentric orbit with $e=0.11 \pm 0.03$. For the remainder of the text, we continue under the assumption of an eccentric orbit, for which we report $P=909 \pm 2 \mathrm{~d}$.

To derive the mass ratio, we also measured the semiamplitude of the sdB RV curve. We fixed the period, eccentricity, and phase to the values determined from the cool companion measurements (the phase was shifted by $180 \mathrm{deg}$ ) and allowed the semi-amplitude and systemic velocity to float freely. Allowing $\gamma$ to adjust accounts for a difference in the surface gravities (gravitational redshifts) of the two components, which results in apparent systemic velocities that differ from one another. In $\mathrm{sdB}+\mathrm{F} / \mathrm{G} / \mathrm{KV}$ systems, this difference can reach upwards of $2 \mathrm{~km} \mathrm{~s}^{-1}$ and was first measured for a hot subdwarf binary by Vos et al. (2012). We find $K_{\mathrm{sdB}}=12.8$ $\pm 1.0 \mathrm{~km} \mathrm{~s}^{-1}$ and $\gamma_{\mathrm{sdB}}=-132.6 \pm 1.1 \mathrm{~km} \mathrm{~s}^{-1}$ for the sdB star. The measured difference in systemic velocities is $\Delta \gamma=2.9 \pm 1.1 \mathrm{~km} \mathrm{~s}^{-1}$. This measurement, when combined with a $\log g$ estimate for the main sequence companion, allows one to compute the gravitational redshift (and thus, $\log g$ ) for the hot subdwarf star, but in this case the errors are too large to make this a fruitful exercise.

From $\mathrm{K}_{\mathrm{MS}}$ and $\mathrm{K}_{\mathrm{sdB}}$, we calculate a mass ratio of $q$ $=0.64 \pm 0.06$, where $q \equiv K_{\mathrm{MS}} / K_{\mathrm{sdB}}$. If we assume $M_{\mathrm{MS}}=1.04 \pm 0.12 \mathrm{M}_{\odot}$ for the cool companion (based on our spectroscopic classification, which assumes solar abundances), we derive a subdwarf mass of $M_{\mathrm{sdB}}=0.66$ $\pm 0.10 \mathrm{M}_{\odot}$. With this precision, we cannot claim a significant deviation from the canonical mass value $(\sim 0.5$ $\left.\mathrm{M}_{\odot}\right)$.

\section{2. $P G 1701+359$}

Heavy dilution of the cool companion's spectral features from the hot subdwarf's continuum made classifying the PG 1701+359 spectrum and measuring velocities more difficult than in the case of PG $1449+653$. Errors on the cool companion RVs ranged from $250 \mathrm{~m} \mathrm{~s}^{-1}$ (HRS) to 1-2 $\mathrm{km} \mathrm{s}^{-1}$ (MRS, MMT). We were not able to derive useful sdB velocities from the He I lines. In view of the expected acceleration, which is much smaller than in the PG $1449+653$ case, they are too weak for our cross-correlation measurement technique to achieve the required precision.

The cool companion RV curve (bottom panel, Figure 1) exhibits a small-amplitude variation $\left(\sim 4 \mathrm{~km} \mathrm{~s}^{-1}\right)$ with a period near 2.0 years. Although an eccentric solution models our data more accurately (naturally), we cannot yet rule out a circular orbit since the best-fitting $e=0.07 \pm 0.04$ solution was rejected by the revised LS test. Instead, we replace our measured value with an upper limit, $e \leq 0.23$, which was determined using their recommended prescription (Appendix 2 of Lucy 2012). The derived orbital parameters from the circular and 
non-circular models agree with one another to within the errors. Our current phase coverage is incomplete as nearly all of our velocities are on the ascending portion of the RV curve; additional measurements on the descending slope will greatly improve the eccentricity estimate. We continue under the assumption of $e=0$, for which we report $P=734 \pm 3$.

\section{GALACTIC KINEMATICS}

Both PG $1449+653$ and PG $1701+359$ display relatively large systemic velocities, so we have calculated their Galactic trajectories to see what conclusions can be drawn concerning their kinematic population (halo vs. thick disk vs. thin disk). We also include as a comparison an analysis of the three systems solved in Paper I (PG $1104+243$, PG $1317+123$, \& PG 1338+611), which were found to have less interesting space motions. A kinematical study requires the full set of $6-\mathrm{D}$ phase space coordinates, which we determine from the right ascension $(\alpha)$, declination $(\delta)$, distance $(d)$, systemic velocity $(\gamma)$, and proper motion $\left(\mu_{\alpha} \cos \delta, \mu_{\delta}\right)$. We summarize these input parameters in Table 5 .

We adopt the values of $\gamma$ shown in Table 4 as the systemic velocity inputs $(e=0.11$ solution for PG $1449+653$, $e=0$ solution for $\mathrm{PG} 1701+359)$. Proper motion measurements with errors ranging from 3-11 mas $\mathrm{yr}^{-1}$ were taken from the Fourth U.S. Naval Observatory CCD Astrograph Catalog (UCAC4; Zacharias et al. 2012). The most difficult parameter to determine is the distance. Here we estimate a range of probable line-of-sight distances to each binary from the distance moduli. We assume $T_{\text {eff }}=24000-38000 \mathrm{~K}$ and $R=0.15-0.22 \mathrm{R}_{\odot}$ for the sdB, taking into account conventional correlations between these parameters for hot subdwarfs. For the cool companion, we assume solar abundances and use main sequence temperatures and radii corresponding to our spectral classifications. These values define absolute Visual magnitudes, which were compared to the apparent Visual magnitudes and, after taking into account the measured dilution of the cool companion (\$3.1) and the extinction (Schlegel et al. 1998), provided us with the rough distance estimates shown in Table 5.

We converted positions and motions to Galactocentric coordinates $5, Y, Z)$ and velocities $(U, V, W)$, adopting a Galactocentric distance of $8.5 \mathrm{kpc}$ for the Sun. The resulting $6-\mathrm{D}$ phase space coordinates are presented in Table 6. We also include in this table the velocity component in the direction of Galactic rotation $(\Theta)$, the component towards the center of the Galaxy $(\Phi)$, and the angular momentum $\left(I_{z}\right)$. The space velocities were transformed to the Local Standard of Rest by removing a solar motion of $(10.0,5.3,7.2) \mathrm{km} \mathrm{s}^{-1}$ (Dehnen \& Binnev 1998). We used the orBit6 code developed by Odenkirchen \& Brosche (1992) to calculate the orbits of the binaries in the axisymmetric Galactic potential of Allen \& Santillan (1991). In this model, the disk rotation speed and volume density are $220 \mathrm{~km}$ $\mathrm{s}^{-1}$ and $0.15 \mathrm{M}_{\odot} \mathrm{pc}^{-3}$, respectively. We reconstructed the path of each system over a 10 Gyr period with time steps of $1 \mathrm{Myr}$ in order to obtain a clear picture of the

\footnotetext{
5 Left-handed Cartesian coordinates where $X$ increases from Galactic center to anti-Solar direction and $Z$ increases towards the Galactic North Pole.
}

shape of each orbit. Figure 2 shows the resulting trajectories in the $Y-X, Z-X$, and $Z-\rho$ planes. The last four columns of Table 6 list the apo- and perigalactic distances ( $R_{a}$ and $R_{p}$ respectively), the maximum $Z$ direction excursion from the disk, and the eccentricity $\left(e \equiv\left(R_{a}-R_{p}\right) /\left(R_{a}+R_{p}\right)\right)$, all of which were determined by integrating the orbits.

Figure 2 reveals the orbits of PG $1449+653$ and PG $1701+359$ to be fundamentally different from those of the other systems. Whereas PG 1104+243, PG 1317+123, and PG $1338+611$ exhibit relatively well-behaved, disklike trajectories that never stray far from the Galactic plane (PG $1317+123$ being the most Sun-like), the orbits of PG $1449+653$ and PG $1701+359$ are significantly more eccentric and carry these systems much farther out of the disk. The orbit of PG $1701+359$ shows surprisingly little angular momentum, with a highly-eccentric orbit $(e \sim 0.8)$ bringing it as close as $1.5 \mathrm{kpc}$ to the Galactic center and as far away as $10.6 \mathrm{kpc}$. PG $1449+653$ currently appears to be near apogalacticon. We remind the reader that all of the orbits calculated here represent approximate trajectories; small changes in the initial $6 \mathrm{D}$ phase-space coordinates can significantly change the results, in addition to any encounters with localized regions of higher density in the Galaxy which are not accounted for by ORBIT6.

We employ the method described in the appendix of Grether \& Lineweaver (2007) to calculate the probabilities that each system belongs to the halo $\left(P_{\text {halo }}\right)$, thick dick $\left(P_{\text {thick }}\right)$, or thin disk $\left(P_{\text {thin }}\right)$. This procedure assumes that all stellar systems belong to one of these three subpopulations, and that the Galactic velocities $(U, V, W)$ and metallicities $([\mathrm{Fe} / \mathrm{H}])$ of each subpopulation follow Gaussian distributions defined by the data in Table 4 of Robin et al. (2003). Since we lack precise metallicity estimates for the two systems studied here, we plot these probabilities in Figure 3 over a broad range of metallicities.

The kinematics alone are enough to rule out thin disk membership for both PG $1449+653$ and PG 1701+359. Choosing between the thick disk and halo populations will ultimately require precise measurements of the metallicity. If $[\mathrm{Fe} / \mathrm{H}]$ turns out to be much less than -1.55 $(-1.25)$, PG $1449+653$ (PG 1701+359) would most likely be a member of the Galactic halo and not the thick disk. Vos et al. (2012) report $[\mathrm{Fe} / \mathrm{H}]=-0.58 \pm 0.11$ dex for $\mathrm{PG}$ $1104+243$; using this value and the system's kinematics, we find that there is $\sim 94 \%$ probability it is a member of the thick disk. We cannot assign memberships to PG $1317+123$ and $\mathrm{PG} 1338+611$ at this time using the data currently available.

The only major 3D kinematical study of hot subdwarfs in the Galaxy was carried out by Altmann et al. (2004) on a sample of 114 stars selected primarily from the Hamburg/ESO (HE) and Palomar Green (PG; Green et al. 1986) surveys; some of their targets were compositespectra systems. They found that the vast majority of sdBs are members of the disk, while only a minority $(\sim 15 \%)$ appear to be halo objects. Of the disk population, the thick disk seems to be preferred over the thin disk. We cannot make any definitive claims concerning Galactic membership differences between the composite-spectra systems and the apparently single or short-period sdB binaries at this time, owing to the small 
TABLE 6

Galactocentric 6-D Phase Space Coordinates and Orbital Parameters

\begin{tabular}{|c|c|c|c|c|c|c|c|c|c|c|c|c|c|}
\hline Target & $\begin{array}{c}X \\
{[\mathrm{kpc}]}\end{array}$ & $\begin{array}{c}Y \\
{[\mathrm{kpc}]}\end{array}$ & $\begin{array}{c}Z \\
{[\mathrm{kpc}]}\end{array}$ & $\begin{array}{c}U \\
{\left[\mathrm{~km} \mathrm{~s}^{-1}\right]}\end{array}$ & $\begin{array}{c}V \\
{\left[\mathrm{~km} \mathrm{~s}^{-1}\right]} \\
\end{array}$ & $\begin{array}{c}W \\
{\left[\mathrm{~km} \mathrm{~s}^{-1}\right]}\end{array}$ & $\left.\begin{array}{c}\Phi \\
{\left[\mathrm{km} \mathrm{s}^{-1}\right]}\end{array}\right]$ & $\begin{array}{c}\Theta \\
{\left[\mathrm{km} \mathrm{s}^{-1}\right]} \\
\end{array}$ & $\begin{array}{c}I_{z} \\
{\left[\mathrm{kpc} \mathrm{km} \mathrm{s}^{-1}\right]}\end{array}$ & $\begin{array}{c}R_{a} \\
{[\mathrm{kpc}]}\end{array}$ & $\begin{array}{c}R_{p} \\
{[\mathrm{kpc}]}\end{array}$ & $\begin{array}{l}z_{\max } \\
{[\mathrm{kpc}]}\end{array}$ & $e$ \\
\hline PG $1449+653$ & -8.67 & +0.64 & +0.73 & -78 & +101 & -81 & +10 & +95 & -829 & 2.61 & 9.91 & 3.73 & 0.58 \\
\hline PG $1701+359$ & -8.21 & +0.46 & +0.41 & -132 & +68 & +94 & +11 & +60 & -492 & 1.48 & 10.69 & 4.70 & 0.76 \\
\hline PG $1104+243$ & -8.61 & -0.07 & +0.30 & -59 & +168 & -51 & +9.9 & +168 & -1447 & 5.25 & 9.37 & 1.12 & 0.28 \\
\hline PG $1317+123$ & -8.42 & -0.05 & +0.31 & +11 & +219 & +46 & +8.7 & +219 & -1843 & 8.23 & 8.88 & 0.87 & 0.04 \\
\hline PG $1338+611$ & -8.56 & +0.14 & +0.22 & +62 & +216 & +72 & +7.9 & +210 & -1797 & 6.96 & 11.16 & 2.21 & 0.23 \\
\hline
\end{tabular}
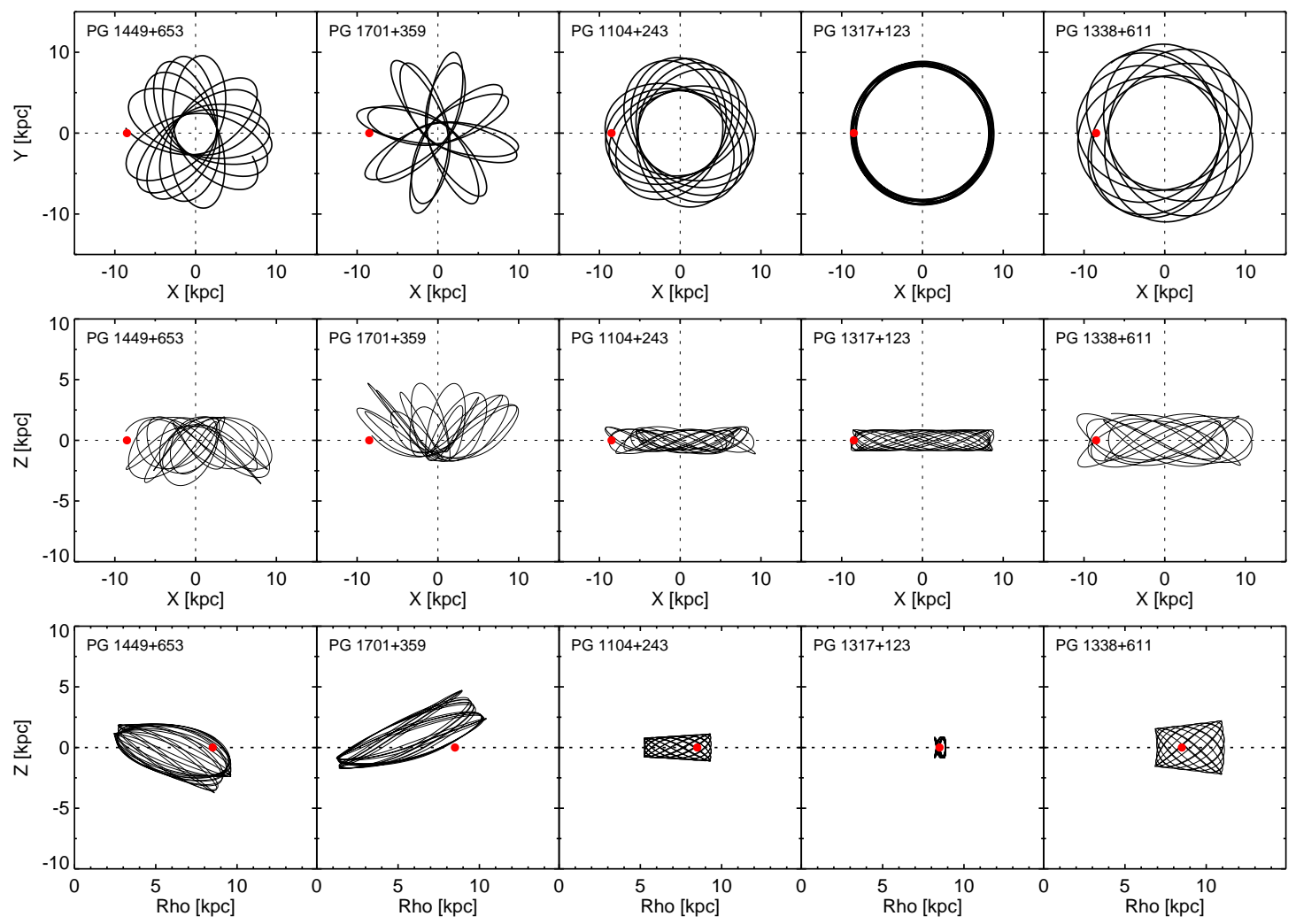

FIG. 2.- Galactocentric orbits for PG 1449+653, PG 1701+359, and the three binaries solved in Paper I, shown over a 10 Gyr period. Orbits were calculated using the potential of Allen \& Santillan (1991). The top two rows show the orbits in the $Y$ - $X$ and $Z$ - $X$ planes, while the bottom row shows the height above the disk $(Z)$ as a function of Galactocentric distance (Rho). In each panel, the location of the Sun is marked with a solid red circle, for reference. (A color version of this figure is available in the online journal.)

number of long-period systems currently studied in sufficient detail. Eventually, a kinematical analysis of a larger sample of composite-spectra binaries, aided by precise proper motion measurements from Gaia, might provide additional insight into the formation scenarios of long-period binaries. As some of these binaries show peak-to-peak RV amplitudes in excess of $15 \mathrm{~km} \mathrm{~s}^{-1}$, it is imperative that such studies use the true systemic velocity when computing Galactic trajectories, as determined from a well-sampled RV curve with full phase coverage.

\section{CONCLUDING REMARKS}

We have presented follow-up spectroscopic observations of the $\mathrm{sdB}+\mathrm{G} / \mathrm{K}$ binaries $\mathrm{PG} 1449+653$ and $\mathrm{PG}$ $1701+359$, the tenth and eleventh such systems for which orbital parameters have been determined. Combining spectroscopic data from the MMT and HET, we find orbital periods around two years for both binaries. While we cannot claim an eccentric orbit in the case of $\mathrm{PG}$ $1701+359$, PG $1449+653$ appears to have a mildly eccentric orbit, thereby joining PG $1338+611$ (Paper I) and possibly PG 1018+243 (Deca et al. 2012) in an emerging group of long-period sdB+/F/G/K binaries with noncircular orbits. If wide sdB binaries continue to show eccentric orbits, this would suggest one of the following possibilities: (1) these systems are or were hierarchical triple-star systems, (2) circularization was never achieved because the sdB progenitor did not fill its Roche lobe, or (3) one of several possible mechanisms pumped eccentricity into the system. Further details concerning eccentric orbits of $\mathrm{sdB}+\mathrm{F} / \mathrm{G} / \mathrm{K}$ binaries and the implications thereof are discussed in Paper I. A kinematical analysis inspired by the relatively large systemic velocities found for each system shows that they are probably not members of the Galactic thin disk; we cannot yet distinguish between thick-disk or halo memberships. 


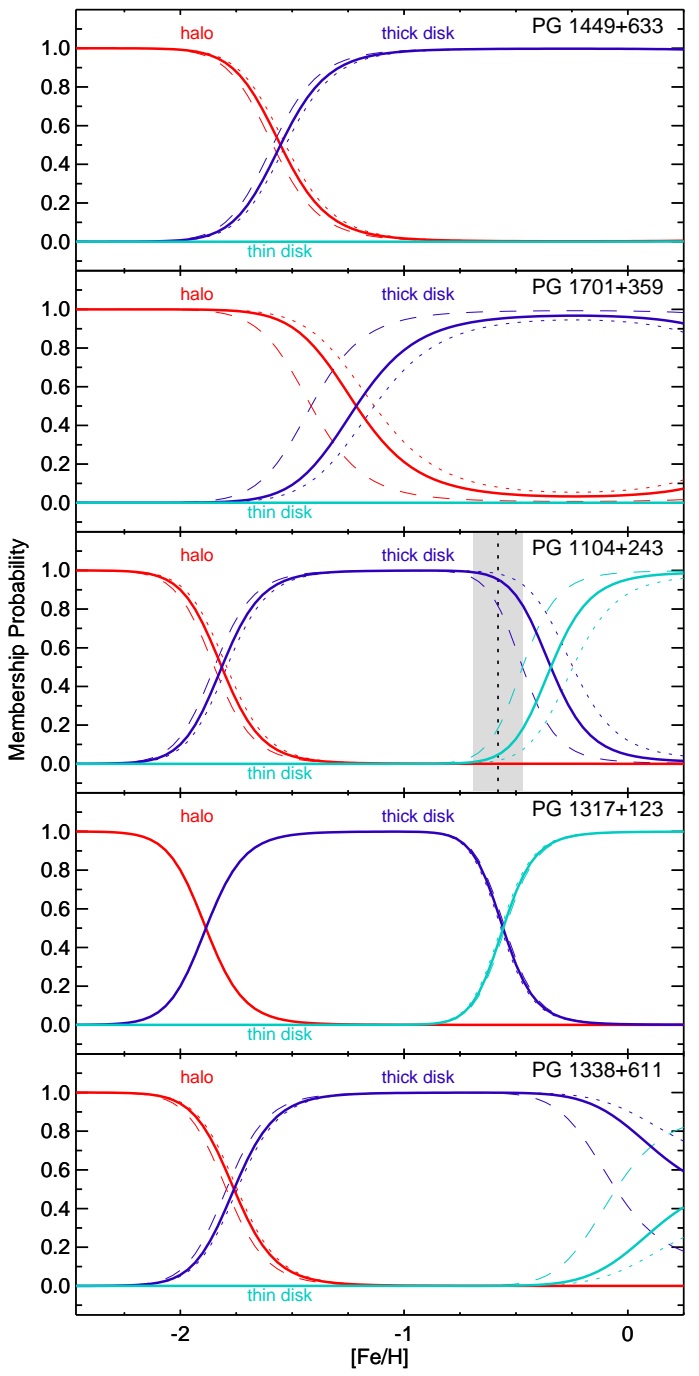

FIG. 3.- Galactic membership probabilities for PG $1449+653$ and PG 1701+359 (top two panels). The solid red, dark blue, and light blue lines denote the probabilities that each system is a member of the halo, thick disk, and thin disk, respectively, for a range of metallicities, using our best estimate for the distance. The dotted and dashed lines show how these probabilities change when we modify the distance to accommodate the uncertainties shown in Table 5. The kinematics alone show it is unlikely either system is a member of the Galactic thin disk. In the bottom three panels, we present the membership probabilities for the three binaries solved in Paper 1. The vertical line and shaded region in the PG 1104+243 panel represent the $[\mathrm{Fe} / \mathrm{H}]$ measurement and associated uncertainty reported by Vos et al. (2012). (A color version of this figure is available in the online journal.)
Our results continue the trend of finding long periods (on the order of years) for hot subdwarf stars with $\mathrm{G} / \mathrm{K}$-type main sequence companions. Binary population synthesis codes (e.g., Han et al. 2002, 2003) are relatively successful at matching the observed orbital periods of sdBs with $\mathrm{M}$ dwarf or white dwarf companions but struggle to reproduce the long periods found for $\mathrm{sdB}+\mathrm{F} / \mathrm{G} / \mathrm{KV}$ systems. Even though relatively few of these long-period binaries have been studied to date, current observations strongly suggest that (1) the assumptions made in some hot subdwarf formation scenarios should be revised and (2) some of the parameterizations in BPS models be re-tuned. As demonstrated by Clausen et al. (2012), subtle variations in the assumptions about the minimum core mass required for helium ignition, the efficiency of common envelope ejection, the envelope binding energy, the criteria for stable mass transfer, and the amount of mass lost during stable mass transfer can lead to BPS models producing significantly different distributions of orbital properties for hot subdwarf binaries. Orbital parameters for many more $\mathrm{sdB}+\mathrm{F} / \mathrm{G} / \mathrm{KV}$ systems must be measured in order to thoroughly evaluate the predictive success of current BPS models and determine which parameterizations and assumptions must be adjusted to match the empirical period distribution.

This material is based upon work supported by the National Science Foundation under Grant No. AST0908642. We thank Rohit Deshpande and Suvrath Mahadevan for providing HRS/HET spectra of MS stars and Eva Ziegerer for providing a copy of the ORBIT6 code. The Hobby-Eberly Telescope is a joint project of the University of Texas at Austin, the Pennsylvania State University, Stanford University, LudwigMaximilians-Universität München, and Georg-AugustUniversität Göttingen. The HET is named in honor of its principal benefactors, William P. Hobby and Robert E. Eberly. This research has made use of NASA's Astrophysics Data System Bibliographic Services and the SIMBAD database, operated at CDS, Strasbourg, France.

Facilities: HET (MRS, HRS), MMT (Blue Spectrograph)

\section{REFERENCES}

Allen, C., \& Santillan, A. 1991, RevMexAA, 22, 255

Altmann, M., Edelmann, H., \& de Boer, K. S. 2004, A\&A, 414, 181

Barlow, B. N., Wade, R. A., Liss, S. E., Østensen, R. H., \& Van Winckel, H. 2012, ApJ, 758, 58 (Paper I)

Brown, T. M., Bowers, C. W., Kimble, R. A., Sweigart, A. V., \& Ferguson, H. C. 2000, ApJ, 532, 308

Clausen, D., \& Wade, R. A. 2011, ApJ, 733, L42

Clausen, D., Wade, R. A., Kopparapu, R. K., \& O'Shaughnessy, R. 2012, ApJ, 746, 186

Cumming, A., Marcy, G. W., \& Butler, R. P. 1999, ApJ, 526, 890

Deca, J., Marsh, T. R., Østensen, R. H., et al. 2012, MNRAS, 421,2798
Dehnen, W., \& Binney, J. J. 1998, MNRAS, 298, 387

Geier, S., Hirsch, H., Tillich, A., et al.. 2011, A\&A, 530, A28

Green, E. M., Fontaine, G., Hyde, E. A., Charpinet, S., \& Chayer, P. 2006, Baltic Astronomy, 15, 167

Green, R. F., Schmidt, M., \& Liebert, J. 1986, ApJS, 61, 305

Greenstein, J. L., \& Sargent, A. I. 1974, ApJS, 28, 157

Grether, D., \& Lineweaver, C. H. 2007, ApJ, 669, 1220

Han, Z., Podsiadlowski, P., Maxted, P. F. L., \& Marsh, T. R. 2003, MNRAS, 341, 669

Han, Z., Podsiadlowski, P., Maxted, P. F. L., Marsh, T. R., \& Ivanova, N. 2002, MNRAS, 336, 449

Heber, U. 1986, A\&A, 155, 33

Johnson, H. L. 1966, ARA\&A, 4, 193 
Liebert, J., Saffer, R. A., \& Green, E. M. 1994, AJ, 107, 1408 Lucy, L. B. 2012, A\&A, 551, 47

Lucy, L. B., \& Sweeney, M. A. 1971, AJ, 76, 544

Mengel, J. G., Norris, J., \& Gross, P. G. 1976, ApJ, 204, 488

Moni Bidin, C., \& Piotto, G. 2010, Ap\&SS, 329, 19

Napiwotzki, R. 2008, in Astronomical Society of the Pacific Conference Series, Vol. 391, Hydrogen-Deficient Stars, ed. A. Werner \& T. Rauch, 257

Nelemans, G. 2010, Ap\&SS, 329, 25

Odenkirchen, M., \& Brosche, P. 1992, Astronomische Nachrichten, 313, 69

Østensen, R. H., \& Van Winckel, H. 2012, in ASP Conf. Ser. 452, Fifth Meeting on Hot Subdwarf Stars and Related Objects, ed. D. Kilkenny, C. S. Jeffery, \& C. Koen, (San Francisco, CA: ASP), 163

Ramsey, L. W., Adams, M. T., Barnes, T. G., et al. 1998, Proc. SPIE, 3352, 34

Robin, A. C., Reylé, C., Derrière, S., \& Picaud, S. 2003, A\&A, 409,523
Saffer, R. A., Green, E. M., \& Bowers, T. 2001, in ASP Conf. Ser. 226, 12th European Workshop on White Dwarfs, ed. J. L.

Provencal, H. L. Shipman, J. MacDonald, \& S. Goodchild (San Francisco, CA: ASP), 408

Saffer, R. A., Bergeron, P., Koester, D., \& Liebert, J. 1994, ApJ, 432,351

Schlegel, D. J., Finkbeiner, D. P., \& Davis, M. 1998, ApJ, 500, 525

Stark, M. A., \& Wade, R. A. 2003, AJ, 126, 1455

Tonry, J., \& Davis, M. 1979, AJ, 84, 1511

Vos, J., Østensen, R. H., Degroote, P., et al. 2012, A\&A, 548, A6

Wang, X., Sharon, Wright, J. T., Cochran, W., et al. 2012, ApJ 761,46

Wright, J. T., \& Howard, A. W. 2009, ApJS, 182, 205

Zacharias, N., Finch, C. T., Girard, T. M., Henden, A., et al. 2012, VizieR Online Data Catalog, I/322 